\title{
Arachidonic acid pathway alterations in cerebrospinal fluid of dogs with naturally occurring spinal cord injury
}

\author{
Rae L. Russell ${ }^{1,2}$, Jonathan M. Levine ${ }^{1}$, Nick D. Jeffery ${ }^{3}$, Colin Young ${ }^{2}$, Armando Mondragon², Bryan Lee ${ }^{2}$, \\ C. Elizabeth Boudreau', C. Jane Welsh² and Gwendolyn J. Levine ${ }^{4^{*}}$
}

\begin{abstract}
Background: Canine intervertebral disc Therniation causes a naturally-occurring spinal cord injury (SCl) that bears critical similarities to human $\mathrm{SCl}$ with respect to both injury pathomechanisms and treatment. As such, it has tremendous potential to enhance our understanding of injury biology and the preclinical evaluation of novel therapies. Currently, there is limited understanding of the role of arachidonic acid metabolites in canine SCI.

Results: The CSF concentrations of PLA2 and PGE2 were higher in SCl dogs compared to control dogs ( $p=0.0370$ and 0.0273 , respectively), but CSF LCT4 concentration in SCl dogs was significantly lower than that in control dogs $(p<0.0001)$. Prostaglandin E2 concentration in the CSF was significantly and positively associated with increased severity of $\mathrm{SCl}$ at the time of sampling $(p=0.041)$ and recovery 42 days post-injury $(p=0.006)$, as measured by ordinal behavioral scores.

Conclusion: Arachidonic acid metabolism is altered in dogs with $\mathrm{SCl}$, and these data suggest that these AA metabolites reflect injury severity and recovery, paralleling data from other model systems.
\end{abstract}

Keywords: Canine, Neurotrauma, Prostaglandin E2, Phospholipase A2

\section{Background}

Several experimental animal models of spinal cord injury (SCI) have been established, including contusion, laceration, clip compression, and crush in a variety of species [1-3]. These systems generate highly stereotypical injuries and minimize heterogeneity in severity, timing of injury, genetic background, and environmental exposure. While elimination of inter-animal variability likely enhances detection of the effects of putative therapeutic interventions, it does not fully reflect the diverse injury characteristics that complicate naturally-occurring SCI [4].

Canine intervertebral disc herniation (IVDH) causes a naturally-occurring form of SCI that bears critical similarities to human SCI with respect to both injury

\footnotetext{
*Correspondence: GJLevine@cvm.tamu.edu

${ }^{4}$ Department of Veterinary Pathobiology, College of Veterinary Medicine and Biomedical Sciences, Texas A\&M University, TAMU 4467, College Station, TX 77843, USA

Full list of author information is available at the end of the article
}

pathomechanisms and treatment. The resulting SCI occurs spontaneously, consists of varying components of compression and contusion, and is treated with a combination of decompressive surgery and physical rehabilitation [5, 6]. Histologic facets of injury parallel those detected in both humans with SCI and SCI models, including axon destruction demyelination, and centrally-oriented necrosis/cavitation [7]. Spinal cord lesions in affected dogs contain activated microglia [8]; have aberrantly increased expression of IL-6, IL-8 [9], and matrix metalloproteinase-9 (MMP-9) [10]; and contain a population of peripherally-derived leukocytes $[8$, 11]. Additionally, these inflammatory events result in loss of blood-spinal cord barrier integrity and increased oxidative stress [12]. The similarities between human SCI and canine SCI resulting from IVDH have prompted the development of validated outcome measures including ordinal gait scores, kinematics, kinetics, urodynamics, and sensory testing in order to detect subtle 
improvement associated with experimental therapeutic interventions [13-15]. Furthermore, studies by several independent groups have utilized dogs with IVDH as a second species to evaluate neuroprotective and potential regenerative strategies headed for human clinical trials $[5,14,16,17]$.

The arachidonic acid (AA) pathway is a critical mediator of secondary SCI and an attractive target for pharmacologic interventions. Metabolism of AA is ubiquitous; this polyunsaturated fatty acid is released from cell membranes by phospholipase A2 (PLA2, for review see Schaloske and Dennis [18]). In rodent spinal cord contusion models, PLA2 protein expression is induced within minutes of injury, persists for up to 7 days post-injury, and correlates with the development of demyelination and neuronal necrosis [19]. Following its release from the cell membrane, AA is metabolized into leukotrienes (LTs) and prostaglandins (PGs) via 5-lipoxygenase (5-LOX) and cyclooxygenase (COX), respectively [20, 21]. Leukotrienes and PGs function as immune cell chemoattractants, vasodilators, inducers of oxidative stress, and modulators of neurosensory processing. Further, LTs and PGs are increased acutely after experimental SCI and remain aberrantly elevated for months post-trauma [22]. Chronic dysregulation of 5-LOX and COX pathways following experimental spinal cord contusion results in depletion of lipid metabolites, altered amino acid biosynthesis, and pro-inflammatory events. Limited investigation of these pathways has occurred in large animal models of SCI. In one study that utilized an experimental canine model of compression/contusion, LTs and PGs were increased within the cerebrospinal fluid (CSF) 1 day following injury and remained elevated for approximately 7 days after the primary event [23].

In this study, we assessed alterations of AA metabolism after SCI in dogs with IVDH by using enzyme-linked immunosorbent assays (ELISA) to measure CSF concentrations of PLA2, leukotriene C4 (LTC4) and prostaglandin E2 (PGE2). We selected these mediators because they represent critical nodes in the AA pathway that are altered in experimental SCI. Our primary objective in this exploratory study was to determine if there were higher concentrations of AA metabolites in the CSF of dogs with SCI than in healthy control dog CSF. Our secondary objective was to determine if, in dogs with $\mathrm{SCI}$, CSF AA pathway metabolites were correlated with functional deficits at the time of sampling and 42-day postinjury recovery as measured by a validated ordinal score.

\section{Methods}

\section{Sample size determination}

Sample size was determined a priori and was based on previous studies that examined inflammatory mediators (e.g., IL-8, C-reactive protein, MMP-9) in the CSF of dogs with IVDH-associated SCIs compared to CSF of healthy control dogs $[24,25]$. In those studies, samples from 8 to 21 healthy controls and 35-47 dogs with IVDH were used to identify significant inter-group differences. Based on these data, we elected to utilize all control $(n=21)$ and SCI samples $(\mathrm{n}=44)$ available within our biobank.

\section{Inclusion and exclusion criteria}

A repository of CSF aliquots collected from the cerebellomedullary cistern, stored at $-80{ }^{\circ} \mathrm{C}$, and housed at Texas A\&M University since December 2009 was screened in February 2014 for samples from dogs with IVDH-associated SCI that met the following inclusion criteria: (1) lesion between T3 and L5 vertebrae; (2) neurologic impairment of $<7$ days duration; (3) surgical decompression of IVDH with post-operative rehabilitation; and (4) complete medical records including neurologic score at admission and follow-up scoring at day 42 post-surgery. Dogs that were part of on-going clinical trials or had a myelogram performed as part of pre-operative diagnostics were excluded from this study.

Healthy control CSF samples were collected from purpose-bred dogs with normal physical and neurologic exams, normal complete blood counts, and normal serum biochemical analysis. All CSF samples from healthy control dogs were collected and stored in the same manner as described for dogs with IVDH-associated SCI and were required to have a normal total nucleated cell count (TNCC) $(<5$ cells $/ \mu \mathrm{L})$ and total protein concentration $(<35 \mathrm{mg} / \mathrm{dL})$.

\section{Sample collection and therapeutic procedures}

Procedures in dogs with naturally-occurring SCI were performed with owner consent and consisted of standard medical and surgical care. Purpose-bred dogs were obtained and used with approval from the Texas A\&M University Animal Care and Use Committee (AUP 2007115; AUP 2011-145). All studies adhered to the National Institutes of Health Guide for Care and Use of Laboratory Animals.

Dogs with SCI underwent complete physical examination, neurologic examination, complete blood count, and serum biochemistry prior to anesthesia. Data including age, gender, duration of SCI, and recent delivery of non-steroidal anti-inflammatory drugs (NSAIDs) or glucocorticoids (GCs) were collected (Table 1). Dogs were considered treated with these drugs if NSAIDs or GCs were administered within the 7-day period prior to CSF collection. Dogs were then pre-medicated with glycopyrrolate (Robinul-V, West-Ward, Eatontown, NJ, USA) and oxymorphone (Numorphan, Endo Pharmaceuticals, Chadds Ford, PA, USA) or hydromorphone (West-Ward, 


\begin{tabular}{|c|c|c|}
\hline Variable & $\begin{array}{l}\text { SCl dogs } \\
(n=44)\end{array}$ & $\begin{array}{l}\text { Healthy control } \\
\text { dogs }(n=21)\end{array}$ \\
\hline \multicolumn{3}{|l|}{ Dogs } \\
\hline Median age & 5.75 years & 3 years \\
\hline MFS at admission & 2.5 & N/A \\
\hline TSCIS at admission & 6 & N/A \\
\hline Injury to anesthesia time & $36.5 \mathrm{~h}$ & N/A \\
\hline \multicolumn{3}{|l|}{ Sexcharacteristics } \\
\hline Female intact & $4(9 \%)$ & $0(0 \%)$ \\
\hline Female spayed & $18(41 \%)$ & $3(14 \%)$ \\
\hline Male intact & $8(18 \%)$ & $5(24 \%)$ \\
\hline Male neutered & $14(32 \%)$ & $13(62 \%)$ \\
\hline \multicolumn{3}{|l|}{ Breeds } \\
\hline Dachshund & $34(76 \%)$ & $0(0 \%)$ \\
\hline Labrador retriever & $0(0 \%)$ & $7(33 \%)$ \\
\hline Mixed breed & $4(9 \%)$ & $5(24 \%)$ \\
\hline \multicolumn{3}{|l|}{ Injuries } \\
\hline T12-13 & $6(14 \%)$ & N/A \\
\hline T13-L1 & $13(30 \%)$ & N/A \\
\hline $\mathrm{L} 1-\mathrm{L} 2$ & $5(11 \%)$ & N/A \\
\hline $\mathrm{L} 2-\mathrm{L} 3$ & $8(18 \%)$ & N/A \\
\hline Other thoracic & $8(18 \%)$ & N/A \\
\hline Other lumbar & $4(9 \%)$ & N/A \\
\hline \multicolumn{3}{|l|}{ Treatments } \\
\hline Non-steroidal anti-inflammatory drugs & $17(39 \%)$ & $0(0 \%)$ \\
\hline Glucocorticoids & $14(32 \%)$ & $0(0 \%)$ \\
\hline Both & $3(<1 \%)$ & $0(0 \%)$ \\
\hline
\end{tabular}

Eatontown, NJ, USA). Following pre-medication, dogs were induced with propofol (Rapinovet, Abbott Labs, Chicago, IL, USA) and intubated, and anesthesia was maintained with sevoflurane (SevoFlo, Abbott Labs, Chicago, IL, USA). Thoracolumbar vertebral column imaging was performed either via magnetic resonance imaging (MR) or computed tomography (CT). Cerebrospinal fluid was then collected via needle puncture of the cerebellomedullary cistern with an aliquot saved and stored at $-80{ }^{\circ} \mathrm{C}$ for further analysis. Following CSF collection and diagnostic imaging, a hemilaminectomy was performed to remove herniated, compressive intervertebral disc material and associated hemorrhage from the epidural space. Either gross appearance of the disc material or histopathology was used to confirm the diagnosis of IVDH.

Following surgery, dogs were recovered and provided intravenous fentanyl citrate (Hospira Inc., Lake Forest, IL, USA) analgesia and bladder evacuation if unable to voluntarily void. Twenty-four hours later, physical rehabilitation consisting of supported overland walking, passive range of motion, and standing strength exercises were initiated. Dogs were released to their owner's care after pain control was achieved via oral analgesics (tramadol hydrochloride, Amneal Pharaceuticals, Hauppauge, NY, USA) and urine could be voluntarily voided or the bladder manually expressed. The owners continued physical rehabilitation exercises for 6 weeks post-operatively.

\section{Neurologic scoring}

Two separate ordinal SCI scores were used in this study, and were applied at initial evaluation and at a 42-day post-SCI re-evaluation. The modified Frankel score (MFS), and the Texas Spinal Cord Injury Score (TSCIS) have both been validated previously in dogs with IVDHassociated SCI and have been shown to have excellent inter-rater agreement, correlate well with MRI-based measures of SCI, and predict 42-day post-SCI motor outcome [26]. For both assessment tools, dogs were considered ambulatory if they could rise unassisted and take ten or more steps without falling. Dogs that were nonambulatory had pelvic limb movements evaluated using tail support. Postural reaction scores were determined by supporting the dog in a standing position and placing the dorsum of the paw in contact with the ground. Conscious perception of mild and severe stimuli was evaluated by pinching the interdigital webbing and clamping the nail bed with hemostats, respectively. Pain sensation was considered intact based on demonstration of a behavioral (e.g., orienting to the stimulus, vocalization) or physiological (e.g., tachycardia, tachypnea) response to stimulation.

The MFS was used as a coarse ordinal system to stratify injured dogs into groups that parallel those in the American Spinal Cord Injury Association Impairment Scale (ASIA). The MFS consists of six strata where $0=$ paraplegic with absent pain sensation from the hindquarters; $1=$ paraplegic with pain sensation intact to severe stimuli; $2=$ parapaplegic with intact sensation for mild stimuli; $3=$ non-ambulatory paraparetic; $4=$ ambulatory paraparetic; and $5=$ signs consistent with spinal pain only.

The TSCIS was developed as a more refined system than the MFS and was used for all analyses that did not require stratification into broad functional categories. With this system, individual limbs are assessed independently and given a score based on sensation, gait, and proprioceptive placing. Sensation was scored as $0=$ absent, $1=$ sensation present to severe stimuli, but absent for mild stimuli, and $2=$ sensation intact. Proprioceptive placing was scored as 0 when absent, 1 when delayed (correction to normal posture taking $>2 \mathrm{~s}$ ), and 2 when considered normal. For gait assessment, scores ranged from 0 to 6 for each limb as follows: $0=$ no voluntary movement present when supported; $1=$ intact 
limb protraction with no ground clearance; $2=$ intact limb protraction with inconsistent ground clearance; $3=$ intact limb protraction with consistent ground clearance; $4=$ ambulatory with moderate paresis/ataxia (will fall occasionally); $5=$ ambulatory with mild paresis/ ataxia (does not fall even on slick surfaces); and $6=$ normal gait.

\section{Measurement of AA pathway metabolite concentrations}

Cerebrospinal fluid concentrations of PLA2, LTC4, and PGE2 were measured using commercially available ELISA (MyBioSource, San Diego, CA). The PLA2 ELISA (for the lipoprotein-associated isoform), catalog \# MBS015390, was performed following the manufacturer's protocol, using a standard curve ranging from 800 to $25 \mathrm{ng} / \mathrm{mL}$. The LTC4 and PGE2 ELISAs (catalog \#MBS013956 and MBS705363, respectively) were also performed following the manufacturer's protocol, and the standard curves used were $31.2-1000 \mathrm{pg} / \mathrm{mL}$ for LTC4 and 31.25-2000 pg/mL for PGE2. All control CSF samples were run as technical duplicates. A single run was performed on injured dogs samples, because volume available was limited.

\section{Statistics}

The primary objective of this study was to determine whether the concentrations of the AA pathway metabolites in CSF are associated with functional recovery status at 42 days. These relationships were explored first by using univariable linear regression with TSCIS at 42 days as the outcome (dependent variable). Multivariable linear regression was then used to examine the effects of the various possible interactions including initial injury severity, time delay between injury and sampling, NSAID and GC administration, through their inclusion as covariates. All analyses were conducted using commercially available software (Stata 11, StataCorp, College Station, TX).

Secondary objectives of this study were to explore relationships between injury and AA metabolite concentrations. We compared the CSF metabolite concentrations between control and SCI dogs using Mann-Whitney tests. Association between AA metabolite concentration and cell count in the CSF and with SCI severity at presentation were analyzed using linear regression. Figures were generated using GraphPad Prism, version 6.0 (GraphPad Software, San Diego, CA).

\section{Results}

\section{Population characteristics}

There were 44 dogs in the SCI group (Table 1). The median age was 5.75 years (range $1-12$ years). The 3 most common breeds were dachshunds $(\mathrm{n}=34 ; 76 \%)$, mixed breeds $(\mathrm{n}=4 ; 9 \%)$, and shih tzus $(\mathrm{n}=3 ; 7 \%)$. There were 4 intact females (9\%), 18 spayed females (41\%), 8 intact males (18\%), and 14 neutered males (32\%). The median duration between the time of initial injury and CSF collection was $36 \mathrm{~h}$ (range 3-182 h). The median MFS before CSF acquisition was 2.5 (a score indicating non-ambulatory paraparesis; scores ranged from 0 to 5 ). The median TSCIS sub-scores at presentation were as follows: nociception 4 (range $0-4$ ), proprioceptive placing 0 (range $0-3$ ), and motor 2 (range $0-10$ ). The most common vertebral levels at which compressive/contusive lesions were located based on $\mathrm{MR}$ and $\mathrm{CT}$ imaging included: T12-T13 $(\mathrm{N}=6 ; 14 \%), \mathrm{T} 13-\mathrm{L} 1(\mathrm{~N}=13$; $30 \%), \mathrm{L} 1-\mathrm{L} 2(\mathrm{~N}=5 ; 11 \%)$, and $\mathrm{L} 2-\mathrm{L} 3(\mathrm{~N}=8 ; 18 \%)$; there were eight dogs with thoracic injuries at other levels $(18 \%)$ and four with lumbar injuries at other levels (9\%). There were 17 dogs (39\%) that received NSAIDs, 14 that received glucocorticoids ( $32 \%)$, and 3 dogs $(<1 \%)$ that received both NSAIDs and glucocorticoids.

The 21 control dogs had a median age of 3 years (range 1-4). The 2 most common breeds were Labrador retriever and beagle (33 and $24 \%$, respectively, Table 1 ). There were 0 intact females, 3 spayed females (14\%), 5 intact males (24\%), and 13 neutered males (62\%). No control dogs received glucocorticoids or NSAIDs.

\section{CSF analysis in dogs with $\mathrm{SCl}$ and control dogs}

In the SCI group, the median TNCC was 2 cells $/ \mu \mathrm{L}$ (range $0-107$ cells $/ \mu \mathrm{L}$ ), the median red blood cell count (RBC) was 10 cells $/ \mu \mathrm{L}$ (range $0-11,005$ cells $/ \mu \mathrm{L}$ ) and the median total protein concentration was $18 \mathrm{mg} / \mathrm{dL}$ (range 9-94 mg/dL). Twelve dogs had pleocytosis $($ TNCC > 5 cells $/ \mu \mathrm{L})$; of these the median percentage of neutrophils was $49 \%$ (range 0-85\%), monocytes $24.5 \%$ (range 0-100 \%), lymphocytes $12 \%$ (range 0-63\%), and eosinophils was $0 \%$ (range $0-3 \%$ ). No pleocytosis was detected in control CSF samples. In the control group, the median TNCC was 0 cells $/ \mu \mathrm{L}$ (range $0-2$ cells $/ \mu \mathrm{L}$ ), the median $\mathrm{RBC}$ was 3 cells $/ \mu \mathrm{L}$ (range $0-730$ cells $/ \mu \mathrm{L}$ ) and the median total protein was $26 \mathrm{mg} / \mathrm{dL}$ (range $10-35 \mathrm{mg} / \mathrm{dL}$ ).

\section{AA pathway mediators are dysregulated in the $\mathrm{CSF}$ of $\mathrm{SCI}$ dogs}

There was significantly higher CSF PLA2 concentration $(\mathrm{p}=0.0370)$ in dogs with SCI (median $=158.65 \mathrm{ng} /$ $\mathrm{mL}$, range $129.47-219.45 \mathrm{ng} / \mathrm{mL}$ ) compared to control dogs (Fig. 1a, median $=140.08 \mathrm{ng} / \mathrm{mL}$, range $106.04-$ $347.93 \mathrm{ng} / \mathrm{mL}$ ). The concentration of LCT4 in the CSF of SCI dogs $($ median $=148.69 \mathrm{pg} / \mathrm{mL}$, range 71.88 $202.37 \mathrm{pg} / \mathrm{mL})$ was significantly lower $(\mathrm{p}<0.0001)$ than that in control dogs (median $=332.27 \mathrm{pg} / \mathrm{mL}$, range 105.49-579.09 pg/mL) (Fig. 1b). The concentration of PGE2 in the CSF of SCI dogs (median $<31 \mathrm{pg} / \mathrm{mL}$, range $<31-451.07 \mathrm{pg} / \mathrm{mL})$ was significantly $(\mathrm{p}=0.0273)$ greater 

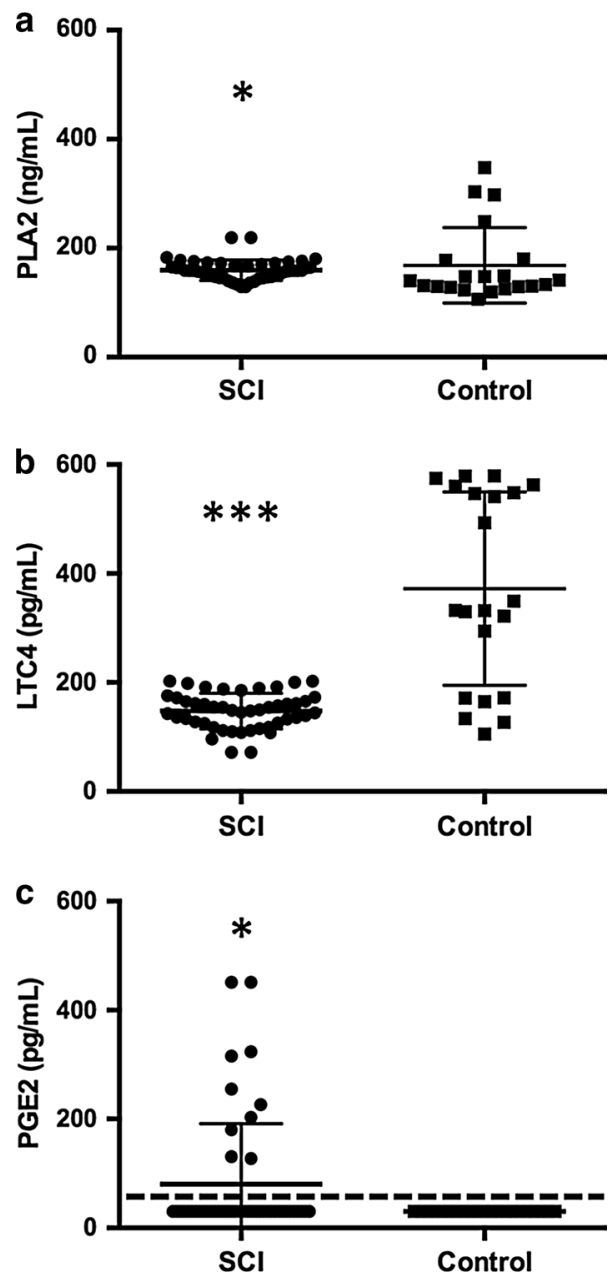

Fig. 1 Scatter plots and line and whisker plots summarizing concentrations of AA metabolites in CSF of 44 dogs with SCl and 21 control dogs. There was a significantly higher CSF PLA2 concentration in dogs with $\mathrm{SCl}$ compared to control dogs (asterisks, $\mathrm{p}=0.0370$ ) (a). The concentration of LCT4 in the CSF of SCl dogs was significantly lower than that in control dogs (asterisks, $\mathrm{p}<0.0001)(\mathbf{b})$. The concentration of PGE2 in the CSF of SCl dogs was significantly higher (c, asterisks, $p=0.0273)$ compared to that in control dogs $(<31 \mathrm{pg} / \mathrm{mL}$, dotted line)

compared to control dogs (Fig. 1c; all control dogs had CSF containing concentrations that were below the limit of detection for this kit $<31 \mathrm{pg} / \mathrm{mL}$ ).

We explored associations between CSF analytes including TNCC, $\mathrm{RBC}$, total protein concentration, and percentage of leukocytes and AA pathway metabolite concentrations in the CSF of SCI dogs. The only significant associations were between PLA2 and TNCC (Fig. 2a, $\mathrm{r}^{2}=0.178$, slope $=-0.400 ; \mathrm{p}=0.004$ ), PGE2 and CSF total protein (Fig. $2 \mathrm{~b}, \mathrm{r}^{2}=0.422$, slope $=3.75$; $\mathrm{p}<0.0001$ ), and PGE2 and CSF RBCs (Fig. $2 \mathrm{c}, \mathrm{r}^{2}=0.451$, slope $=0.370 ; \mathrm{p}<0.0001$ ).
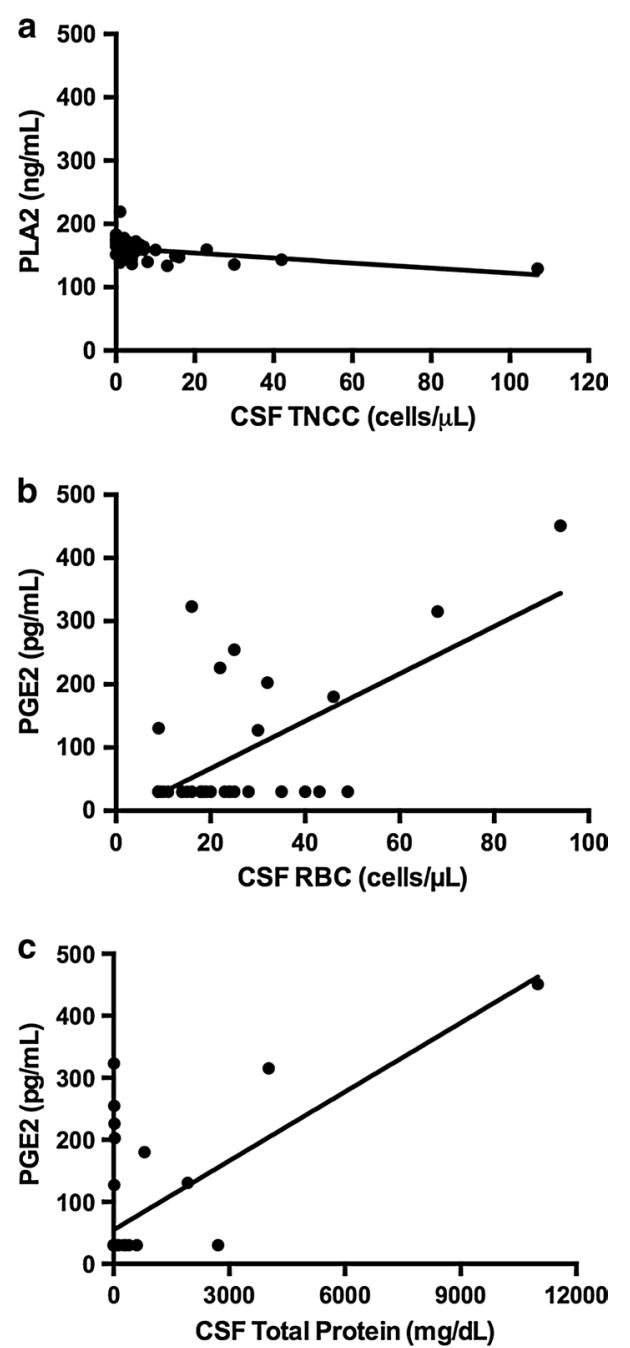

Fig. 2 Linear regressions amongst CSF AA pathway metabolite concentrations and total nucleated cell counts (TNCC, cells/ $\mu \mathrm{L}$ ), CSF total protein ( $\mathrm{mg} / \mathrm{dL}$ ), and CSF red blood cell count (cells/ $\mu \mathrm{L}$ ) in $44 \mathrm{SCl}$ dogs. PLA2 concentration was negatively associated with TNCC in dogs with SCI $\left(r^{2}=0.178\right.$, slope $\left.=-0.400 ; p=0.004\right)(\mathbf{a})$. CSF PGE2 concentration correlated positively with CSF total protein concentration $\left(\mathbf{b}, r^{2}=0.422\right.$, slope $\left.=3.75 ; p<0.0001\right)$, and CSF RBC $\left(\mathbf{c}, R^{2}=0.451\right.$, slope $\left.=0.370 ; p<0.0001\right)$

\section{CSF PGE2 is correlated with $\mathrm{SCl}$ severity and 42-day outcome}

Prostaglandin E2 concentration in the CSF was significantly and positively associated with increasing severity of SCI at the time of sampling, as measured by the MFS in univariate and multivariate models (Fig. 3c, $\mathrm{p}=0.029$ and $\mathrm{p}=0.041$, respectively). No other AA mediators were associated with SCI severity at the time of sample acquisition (Fig. 3a, b). The CSF concentrations of PLA2 and LTC4 were not significantly associated with 42 day postSCI TSCIS (Fig. 4a, b, $\mathrm{p}=0.970$ and 0.262 , respectively). 

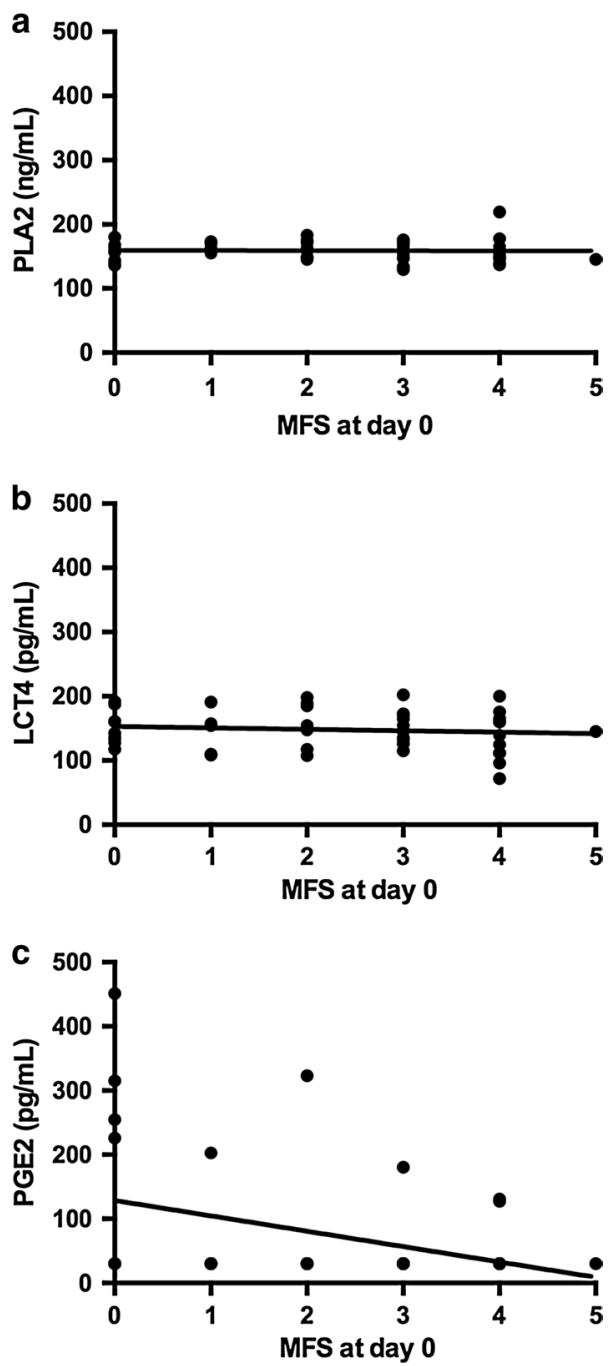

Fig. 3 Linear regressions of CSF AA pathway metabolites and modified Frankel scores (MFS) at day of hospital admission (day 0). PLA2 and LTC4 were not significantly correlated to MFS at day $0\left(r^{2}=0.0004\right.$, slope $=-0.221 ; p=0.894$, and $r^{2}=0.012$, slope $=0 .-2.23 ; p=0.4756$, respectively) (a, b). PGE2 was higher in $\mathrm{SCl}$ dogs with lower MFS at day $0\left(r^{2}=0.137\right.$, slope $=-23.9$; $\mathrm{p}=0.013)(\mathbf{c})$

Prostaglandin E2 concentration was significantly and negatively associated with 42-day post-SCI recovery as measured by the TSCIS in univariate and multivariate models (Fig. 4c, $\mathrm{r}^{2}=0.199$, slope $=-9.61, \mathrm{p}=0.003$ and $p=0.006$, respectively). Because of the low number of SCI dogs in which PGE2 reached detectable concentrations we examined the sensitivity of this result to the numerous null values by repeating the test but including only the dogs with detectable values; both univariable $\left(\mathrm{r}^{2}=0.39\right.$, slope $\left.=-10.47, \mathrm{p}=0.073\right)$ and multivariable $\left(\mathrm{r}^{2}=0.51\right.$, slope $\left.=-1.77, \mathrm{p}=0.137\right)$ analysis revealed a
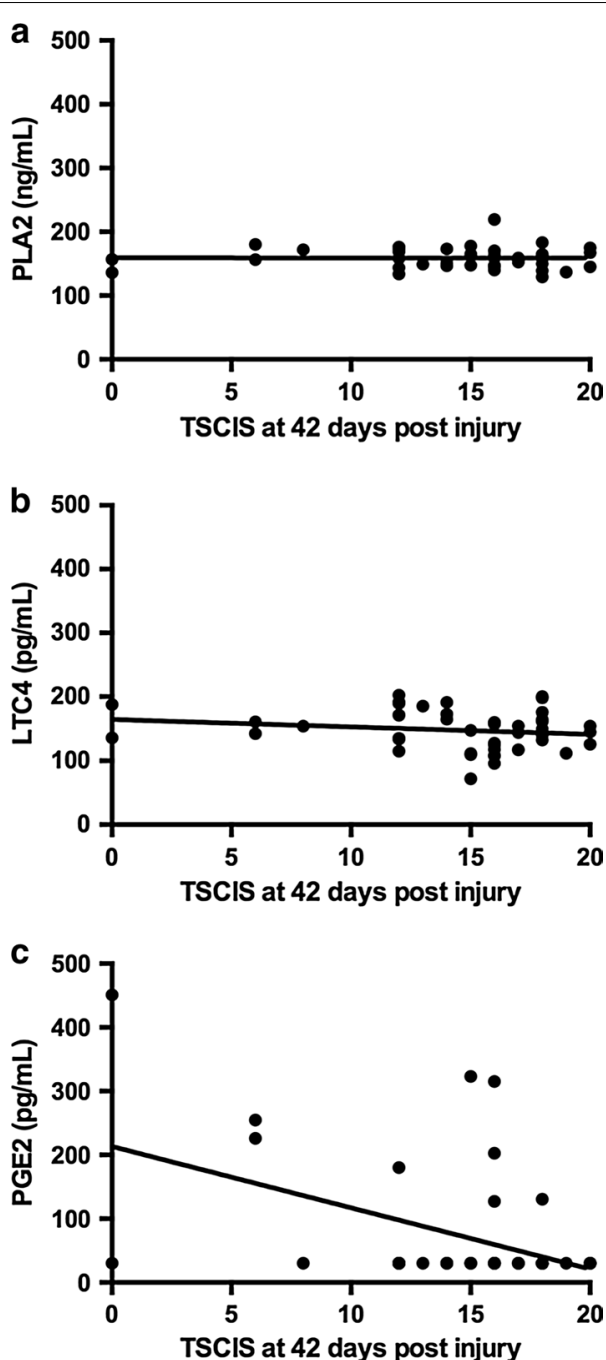

Fig. 4 Linear regressions of AA pathway metabolites and Texas Spinal Cord Injury Scores (TSCIS) at day 42 post-injury. PLA2 and LTC4 were not significantly correlated to TSCIS at day $42\left(r^{2}=0.00003\right.$, slope $=-0.021 ; p=0.970$, and $r^{2}=0.030$, slope $=0 .-1.166$; $p=0.262$, respectively) (a, b). PGE2 was significantly correlated to lower TSCIS at day 42 in dogs with SCI $\left(r^{2}=0.199\right.$, slope $=-9.62$; $\mathrm{p}=0.002)(\mathbf{c})$

non-significant association, although this may also result from the much reduced power of these tests.

\section{Discussion}

This study broadly compared measures of AA metabolism in dogs after naturally-occurring SCI with that in healthy control dogs. The concentration of PLA2, which frees AA from phospholipid membranes, was significantly higher in SCI versus control dogs, and had a weak negative association with the total nucleated cell count in the CSF. The CSF concentration of LTC4, a 
pro-inflammatory leukotriene, was significantly lower in dogs with naturally-occurring SCI compared to control dogs. The CSF concentration of PGE2 was significantly higher in SCI dogs compared to control dogs, and significant relationships existed between CSF PGE2 concentration, initial SCI severity, as well as 42-day post-SCI recovery.

Here, we found CSF concentration of lipoproteinassociated PLA2 was significantly higher in SCI dogs compared to control dogs. In studies performed on rodent spinal cord homogenates post-SCI, the secretory isoform of PLA2 increases within hours of injury, is over-expressed for days following $\mathrm{SCI}$, and is negatively correlated with recovery of locomotion [20]. Critically, there are 27 isoforms of PLA2, of which only 7 have been clearly demonstrated to be dysregulated in SCI [20]. We chose to measure lipoprotein-associated PLA2 because it has not been previously evaluated in the context of SCI, up-regulation has been established in neuro-inflammatory diseases such as Alzheimer's [27], it is secreted from inflammatory cells known to be present within injured cords, and validated methodologies existed to measure it in dogs. While findings here suggest lipoprotein-associated PLA2 is released following injury, there was no association between CSF PLA2 concentration and injury severity at the time of sampling or 42-day post-SCI outcome. Additionally, CSF PLA2 concentration was weakly, but negatively, associated with CSF TNCC. The complex and overlapping role of PLA2 isoforms, treatment of dogs with immune-modulating drugs, and sample size may explain our inability to detect associations between CSF lipoprotein-associated PLA2 concentrations and certain facets of injury.

The CSF concentration of LTC4 was significantly higher in healthy control dogs compared to those with SCI. This finding is in contrast to data from a guinea pig spinal cord contusion model, which showed increased parenchymal LTC4 $10 \mathrm{~min}$ after SCI that persisted $60 \mathrm{~min}$ post-SCI. Data from dogs with experimental spinal cord contusion likewise showed abrupt, early increases in LTC4 concentration, measured within the CSF [23]. Our results may have differed from these previous studies for a variety of reasons. First, the median time between SCI and CSF sampling in our population was $36 \mathrm{~h}$; thus, we may not have captured many dogs with post-injury elevations in LTC4. Secondly, a proportion of the naturally injured dogs studied here received either NSAIDs or glucocorticoids, both of which could reduce LT production. Additionally, post-SCI increases in CSF or parenchymal LTC4 may be species-specific, or model-specific. In a study of cats with experimental compressive SCI, LTC4 concentration was not significantly different between sham and SCI animals [28]. Finally, we believe post-SCI shunting within the LT pathway could be possible and might explain the higher CSF LTC4 concentration in control dogs compared to those with injury in this study. For example, macrophages (the predominant inflammatory cell that releases LTC4) that are exposed to a proinflammatory environment in vitro have reduced LTC4 synthase mRNA expression [29].

Cerebrospinal PGE2 concentration was significantly increased in dogs with SCI compared to healthy control dogs. Additionally, CSF PGE2 concentration was significantly and positively associated with CSF protein concentration and RBC in injured dogs; both these CSF analytes are increased as a result of intrathecal bleeding and blood-spinal cord barrier disruption [30]. Finally, CSF PGE2 concentration was significantly and positively associated with more severe injury at the time of sampling and also 42-day post-SCI recovery as measured by an ordinal scoring system. The relationship between CSF PGE2 and 42-day post-SCI recovery was assessed using multivariate logistic regression. The strength of this approach is that it takes account of other contributory factors such as immunomodulatory drug administration, initial injury severity, and timing of injury. When we assessed associations between CSF PGE2 and 42-day outcome only using dogs with SCI that had detectable CSF PGE2, the relationships were non-significant. While examining relationships in this manner does eliminate bias from null values, it substantially reduces statistical power (nine dogs assessed). Biologic facets of SCI and recovery are typically investigated in rodent contusion models under a series of highly controlled conditions. Here, we assessed AA pathway metabolites in a naturallyoccurring, large animal model of SCI that recapitulates many features of human injury. There are limitations inherent to utilizing samples from dogs with naturally occurring SCI, one of which is the administration of immune response-modulating drugs prior to sample collection. Multivariate logistic regression was used to mitigate influence of administration of these drugs when assessing relationships between CSF AA metabolite concentration and 42-day outcome. We did not, however, directly examine the impact of prior NSAID or GC delivery on CSF AA metabolite concentration in injured dogs. This study could not be adequately powered to investigate the influence of these drugs because of the great variety of interactions between time of injury, time of drug administration, and time of sample collection. Certainly, it is possible that interactions between these drugs and targets in the AA pathway impacted data reported here. Additionally, heterogeneity in injury severity, timing of injury, and vertebral level of compression that are inherent to clinical studies can affect the ability to detect significant inter-group differences. Despite these 
limitations, this study suggests that lipoprotein associated PLA2, LTC4, and PGE2 are all associated with SCI and may provide information relevant to recovery of function. These data, combined with those from other model systems, provide further evidence that AA metabolites are a viable target for pre-clinical SCI trials.

\section{Conclusion}

We assessed three mediators of AA metabolism in the CSF of dogs with SCI and found evidence that PLA2 and PGE2 concentration is altered during injury and PGE2 concentration is associated with SCI severity and recovery. Previous studies in other neurotrauma model systems likewise have suggested that PLA2 and PGE2 exacerbate SCI. Additionally, we found decreased CSF LTC4 concentration in dogs with SCI compared to healthy dogs, which is contrary to what has been described in experimental models. Interspecies differences in inflammatory networks, variability in injury severity and the anatomic level of injury, and assessment of CSF rather than parenchyma may have influenced the results of this study. Future studies using larger populations of injured dogs to confirm findings in this study and more broadly screen AA pathway constituents might provide valuable information, as could quantification of pathway enzyme mRNA.

\begin{abstract}
Abbreviations
SCl: spinal cord injury; CSF: cerebrospinal fluid; PLA2: lipoprotein-associated phospholipase A2; LTC4: leukotriene C4; PGE2: prostaglandin E2; IVDH: intervertebral disc herniation; MMP-9: matrix metalloproteinase-9; AA: arachidonic acid; LTs: leukotrienes; PGs: prostaglandins; 5-LOX: 5-lipoxygenase; COS: cyclooxygenase; ELISA: enzyme-linked immunosorbent assay; TNCC: total nucleated cell count; NSAIDs: non-steroidal anti-inflammatory drugs; GCs: glucocorticoids; MR: magnetic resonance imaging; CT: computed tomography; MFS: modified Frankel score; TSCIS: Texas Spinal Cord Injury Score; ASIA: American Spinal Cord Association Impairment Score.
\end{abstract}

\section{Authors' contributions}

RR and JL drafted the manuscript. NJ performed the statistical analysis. AM and BL carried out the immunoassays. CEB provided critical manuscript revising. JL, CJW, CY, and GL conceived of the study, and participated in its design and coordination and helped to draft the manuscript. All authors read and approved the final manuscript.

\section{Author details}

${ }^{1}$ Department of Small Animal Clinical Sciences, College of Veterinary Medicine and Biomedical Sciences, Texas A\&M University, College Station, TX 77843, USA. ${ }^{2}$ Department of Veterinary Integrative Biosciences, College of Veterinary Medicine and Biomedical Sciences, Texas A\&M University, College Station, TX 77843, USA. ${ }^{3}$ Department of Veterinary Clinical Sciences, College of Veterinary Medicine, lowa State University, 1720 Veterinary Medicine, Ames, IA 50011, USA. ${ }^{4}$ Department of Veterinary Pathobiology, College of Veterinary Medicine and Biomedical Sciences, Texas A\&M University, TAMU 4467, College Station, TX 77843, USA.

\section{Availability of data and material}

The data used in this study will not be publically shared to protect clientpatient confidentiality.

\section{Competing interests}

The authors declare that they have no competing interests.

\section{Ethics approval and consent to participate}

Animal procedures were performed with informed owner consent and purpose-bred dogs were obtained with approval from the Texas A\&M University Institutional Animal Care and Use Committee (AUP 2007-115; AUP 2011-145).

\section{Funding}

The materials used to perform the study were purchased with start-up funds of GJL and JL.

Received: 16 November 2015 Accepted: 3 June 2016

Published online: 10 June 2016

\section{References}

1. Young W. Spinal cord contusion models. Prog Brain Res. 2002;137:231-55

2. Poon PC, Gupta D, Shoichet MS, Tator CH. Clip compression model is useful for thoracic spinal cord injuries: histologic and functional correlates. Spine. 2007;32(25):2853-9.

3. Guth L, Zhang Z, DiProspero NA, Joubin K, Fitch MT. Spinal cord injury in the rat: treatment with bacterial lipopolysaccharide and indomethacin enhances cellular repair and locomotor function. Exp Neurol. 1994;126(1):76-87.

4. Jeffery ND, Smith PM, Lakatos A, Ibanez C, Ito D, Franklin RJM. Clinical canine spinal cord injury provides an opportunity to examine the issues in translating laboratory techniques into practical therapy. Spinal Cord. 2006:44(10):584-93.

5. Levine JM, Levine GJ, Porter BF, Topp K, Noble-Haeusslein LJ. Naturally occurring disk herniation in dogs: an opportunity for pre-clinical spinal cord injury research. J Neurotrauma. 2011;28(4):675-88.

6. Olby N, Halling KB, Glick TR. Rehabilitation for the Neurologic Patient. Vet Clin North Am Small Anim Pract. 2005;35(6):1389-409.

7. Smith PM, Jeffery ND. Histological and ultrastructural analysis of white matter damage after naturally-occurring spinal cord injury. Brain Pathol. 2006;16(2):99-109.

8. Boekhoff TMA, Ensinger E-M, Carlson R, Bock P, Baumgärtner W, Rohn K, Tipold A, Stein VM. Microglial contribution to secondary injury evaluated in a large animal model of human spinal cord trauma. J Neurotrauma. 2011;29(5):1000-11

9. Spitzbarth I, Bock P, Haist V, Stein VM, Tipold A, Wewetzer K, Baumgartner $\mathrm{W}$, Beineke A. Prominent microglial activation in the early proinflammatory immune response in naturally occurring canine spinal cord injury. Neuropathol Exp Neurol. 2011;70(8):703-14.

10. Bock P, Spitzbarth I, Haist V, Stein VM, Tipold A, Puff C, Beineke A, Baumgärtner W. Spatio-temporal development of axonopathy in canine intervertebral disc disease as a translational large animal model for nonexperimental spinal cord injury. Brain Pathol. 2013;23(1):82-99.

11. Griffiths IR. Some aspects of the pathology and pathogenesis of the myelopathy caused by disc protrusions in the dog. J Neurol Neurosurg Psychiatry. 1972;35(3):403-13.

12. Marquis A, Packer RA, Borgens RB, Duerstock BS. Increase in oxidative stress biomarkers in dogs with ascending-descending myelomalacia following spinal cord injury. J Neurol Sci. 2015;353(1-2):63-9.

13. Hamilton L, Franklin RJ, Jeffery ND. Development of a universal measure of quadrupedal forelimb-hindlimb coordination using digital motion capture and computerised analysis. BMC Neurosci. 2007;8(1):1-11.

14. Granger N, Blamires H, Franklin RJ, Jeffery ND. Autologous olfactory mucosal cell transplants in clinical spinal cord injury: a randomized double-blinded trial in a canine translational model. Brain. 2012;135(Pt 11):3227-37.

15. Olby N, Harris T, Burr J, Muñana K, Sharp N, Keene B. Recovery of pelvic limb function in dogs following acute intervertebral disc herniations. J Neurotrauma. 2004;21(1):49-59. 
16. Baltzer WI, McMichael MA, Hosgood GL, Kerwin SC, Levine JM, Steiner JM, Ruaux CG. Randomized, blinded, placebo-controlled clinical trial of $\mathrm{N}$-acetylcysteine in dogs with spinal cord trauma from acute intervertebral disc disease. Spine. 2008;33(13):1397-402.

17. Lim JH, Muguet-Chanoit AC, Smith DT, Laber E, Olby NJ. Potassium channel antagonists 4-aminopyridine and the T-butyl carbamate derivative of 4-aminopyridine improve hind limb function in chronically non-ambulatory dogs; a blinded, placebo-controlled trial. PLOS ONE. 2014;9(12):e116139.

18. Schaloske RH, Dennis EA. Review: the phospholipase A2 superfamily and its group numbering system. BBA-Mol Cell Biol Lipids. 2006;1761:1246-59.

19. Lopez-Vales R, Ghasemlou N, Redensek A, Kerr BJ, Barbayianni E, Antonopoulou G, Baskakis C, Rathore Kl, Constantinou-Kokotou V, Stephens D, et al. Phospholipase A2 superfamily members play divergent roles after spinal cord injury. FASEB J. 2011;25(12):4240-52.

20. David S, Greenhalgh AD, López-Vales R. Role of phospholipase A2 s and lipid mediators in secondary damage after spinal cord injury. Cell Tissue Res. 2012;349(1):249-67

21. Phillis JW, Horrocks LA, Farooqui AA. Review: cyclooxygenases, lipoxygenases, and epoxygenases in CNS: their role and involvement in neurological disorders. Brain Res Rev. 2006:52:201-43.

22. Dulin JN, Karoly ED, Wang Y, Strobel HW, Grill RJ. Licofelone modulates neuroinflammation and attenuates mechanical hypersensitivity in the chronic phase of spinal cord injury. J Neurosci. 2013;33(2):652-64
23. Nishisho TMD, Tonai TMD, Tamura YMD, Ikata TMD. Experimental and clinical studies of eicosanoids in cerebrospinal fluid after spinal cord injury. Neurosurgery. 1996;39(5):950-7.

24. Taylor AR, Welsh CJ, Young C, Spoor E, Kerwin SC, Griffin JF, Levine GJ, Cohen ND, Levine JM. Cerebrospinal fluid inflammatory cytokines and chemokines in naturally occurring canine spinal cord injury. J Neurotrauma. 2014;31(18):1561-9.

25. Anderson KM, Young C, Welsh CJ, Levine GJ, Kerwin SC, Boudreau CE, Reyes I, Mondragon A, Griffin JF, Cohen ND, et al. Acute phase proteins in cerebrospinal fluid from dogs with naturally occurring spinal cord injury. J Neurotrauma. 2015;32:1658-65.

26. Levine GJ, Levine JM, Budke CM, Kerwin SC, Au J, Vinayak A, Hettlich BF, Slater MR. Description and repeatability of a newly developed spinal cord injury scale for dogs. Prev Vet Med. 2009;89(1-2):121-7.

27. Chalbot S, Zetterberg H, Blennow K, Fladby T, Grundke-lqbal I, Iqbal K. Cerebrospinal fluid secretory Ca2+-dependent phospholipase A2 activity: a biomarker of blood-cerebrospinal fluid barrier permeability. Neurosci Lett. 2010;478(3):179-83.

28. Hall ED, Yonkers PA, Taylor BM, Sun FF. Lack of effect of postinjury treatment with methylprednisolone or tirilazad mesylate on the increase in eicosanoid levels in the acutely injured cat spinal cord. J Neurotrauma. 1995;12(3):245-56.

29. Serio KJ, Luo C, Luo L, Mao JT. TNF-a downregulates the leukotriene C4 synthase gene in mononuclear phagocytes. Am J Physiol Lung Cell Mol Physiol. 2007;292(1):L215-22.

30. Stammers AT, Liu J, Kwon BK. Expression of inflammatory cytokines following acute spinal cord injury in a rodent model. J Neurosci Res. 2012;90(4):782-90.

\section{Submit your next manuscript to BioMed Central and we will help you at every step:}

- We accept pre-submission inquiries

- Our selector tool helps you to find the most relevant journal

- We provide round the clock customer support

- Convenient online submission

- Thorough peer review

- Inclusion in PubMed and all major indexing services

- Maximum visibility for your research

Submit your manuscript at www.biomedcentral.com/submit

C) BioMed Central 